\title{
Impact of Automatic Region-of-Interest Coding on Perceived Quality in Mobile Video
}

\author{
Ivan Himawan • Wei Song • Dian Tjondronegoro
}

Received: date / Accepted: date

\begin{abstract}
At present, the most reliable method to obtain end-user perceived quality is through subjective tests. In this paper, the impact of automatic regionof-interest (ROI) coding on perceived quality of mobile video is investigated. The evidence, which is based on perceptual comparison analysis, shows that the coding strategy improves perceptual quality. This is particularly true in low bit rate situations. The ROI detection method used in this paper is based on two approaches: (1) automatic ROI by analyzing the visual contents automatically, and (2) eye-tracking based ROI by aggregating eye-tracking data across many users, used to both evaluate the accuracy of automatic ROI detection and the subjective quality of automatic ROI encoded video. The perceptual comparison analysis is based on subjective assessments with 54 participants, across different content types, screen resolutions, and target bit rates while comparing the two ROI detection methods. The results from the user study demonstrate that ROIbased video encoding has higher perceived quality compared to normal video encoded at a similar bit rate, particularly in the lower bit rate range.
\end{abstract}

Keywords Mobile video quality · human vision system · video coding $\cdot$ subjective quality

This research was carried out as part of the activities of, and funded by, the Smart Services Cooperative Research Centre (CRC) through the Australian Government's CRC Programme (Department of Innovation, Industry, Science and Research).

Queensland University of Technology

2 George Street Brisbane, QLD, Australia

Tel.: +61-7-31381117

Fax: +61-7-31384438

E-mail: i.himawan@qut.edu.au, w1.song@qut.edu.au, dian@qut.edu.au

\section{Introduction}

Video services such as video-on-demand and video conferencing are rapidly growing with the proliferation of mobile technologies that support ubiquitous video services for a wide variety of applications. Video streaming generally requires a higher and more reliable network bandwidth compared to other media such as text, image, or sound. With the freedom to use mobile video services anytime and anywhere via wireless networks, customers put an ever-increasing amount of pressure on the network and content providers to serve smooth streaming high quality video.

The small viewing size and bandwidth-constrained environments where mobile devices typically operate often result in unpleasant experiences. The video content types, resolution, and bit rates affect user acceptability $[42,30,53]$, and many techniques have been proposed to present visual media on mobile devices effectively such as video retargeting for the purpose of adapting large resolution video to a smaller screen size $[38,40]$.

While watching images or videos, human can only observe a particular region that visually interests or captures their attention. Within that region, only the area around the point of fixation is captured by the eyes at a higher resolution and decreases rapidly in the peripheral regions. A good rule of thumb is that, the fovea, which is responsible for seeing fine details, only sees an area about as large as ones thumb width at arms length or about two degrees of visual angle [47]. This fact is particularly important for optimizing the perceptual quality of a video sequence since viewers will never see the whole screen at one point in time. It is hypothesized that, by prioritizing higher quality in the important regions (at the expense of reduced quality in non-important regions), perceived quality can be im- 
proved while using the same bit rate $[32,37]$. In particular, when the bandwidth is limited, more bits can be allocated to the important regions at the cost of reduced bits in the other regions to achieve acceptable quality. For example, in videoconferencing applications, perceptual quality can be improved by prioritizing the face $[8,28]$, or the face and hand of a person talking in sign language [9]. A similar strategy can be applied to any types of videos, and is not limited to only faces or hands, with the assumption that viewers will most likely notice quality improvement in ROI, and ignore lower quality in other (non-ROI) regions. A survey of perceptual video compression algorithms can be found in [35] and [61].

The video quality on mobile devices has not been extensively researched and there are only limited studies on the quality of videos encoded with ROI as a means to improve the perceptual quality of videos viewed on mobile devices, particularly in low bit rate situations. Table 1 lists the current mobile video quality assessment studies, and only studies such as [53,22] used ROI coding, but such studies were limited only to soccer videos such as in [53] rather than use of videos with various genres, and they did not employ automatic ROI to define the region-of-interest. While understanding and modeling ROI coding under real mobile conditions is the ultimate goals of this study, this article in the current form is limited to the effects of ROI coding on coding bit rates, devices, and contents, and analyzing how these factors affect perceived quality. Studies involving humans are highly subjective in nature and ratings can display dramatic variations depending not only on the stimulus perceived but also the mental state of the subjects [44]. The current user study is designed to ensure uniformity across subjects to eliminate many unpredicted influencing factors (e.g., the reaction of the human visual system to changes in surrounding illumination, user context and motivations, bandwidth connections) to provide more statistically meaningful results.

This paper investigates the use of automatic ROIbased video coding to improve the perceptual quality of mobile videos which is implemented on top of H.264/AVC. Due to the fact that mobile devices have various screen sizes and resolutions, this study considers how ROI should be adapted effectively when it is displayed on the target devices. The ROI is predicted automatically using the bottom-up saliency models for detecting salient image regions and is evaluated using eye-tracking based ROI on mobile devices.

In summary, the contributions of this paper are twofold: (1) Subjective assessment of ROI-based videos viewed on mobile devices across different content types, screen resolutions, target bit rates, and the two ROI detection methods; (2) The use of eye-tracking based ROI by aggregating eye-tracking data across many users to both evaluate the accuracy of automatic ROI detection and the subjective quality of automatic ROI encoded video. Furthermore, the database for evaluation is publicly available and the devices and video resolutions for conducting the human studies are popular in today's market.

The rest of this paper is structured as follows. Section 2 discusses related work. Section 3 describes methods to determine the region of interest automatically and by using eye-tracking data, and is followed by presenting the ROI-based video encoding process. Section 4 presents the testing materials used in the subjective testing and the procedures for subjective assessment. Section 5 presents the results of ROI detection accuracy, visual quality improvement of ROI, subjective testing results, the effect of content type on subject preference, and the estimation of bandwidth saving. The findings are discussed in Section 6, followed by the conclusion in Section 7.

\section{Related Work}

\subsection{Region-of-Interest Detection}

Early work by Koch et al. and subsequent work by Itti et al. $[31,23]$ suggests that visual attention is a result of fast, pre-attentive, bottom-up, data driven processes of saliency detection, which happen in conjunction with slower, task-dependent, top-down, goal driven processes. This biologically inspired model is widely adopted as a theoretical foundation for human attention mechanisms. In the context of ROI coding, there are two main approaches for determining ROI: pixel domain and compressed domain [14].

The pixel domain approach identifies ROI as simple or complex objects that are of interest to specific applications or situations by using pixel domain models such as the visual attention model, object detection model, or face detection model. The visual attention model often focuses on bottom-up processes (known as saliency models) due to its simplicity. Salient features which are known to attract human attention such as colors, intensities, orientations, and motion are used to render certain image regions more conspicuous than their surroundings. Using the object detection model, features that represent objects characteristics are used to construct feature-based representation of objects for successful detection. For example, face detection can be considered as a specific class of object-class detection, which is particularly useful in videoconferencing 
Table 1 Selected examples of recent quality assessments of videos on mobile devices (chronologically ordered).

\begin{tabular}{|c|c|c|c|c|}
\hline Ref & $\begin{array}{l}\text { Baseline } \\
\text { Codec }\end{array}$ & Devices & Test Videos & User Study Experiments \\
\hline ours & H.264/AVC & $\begin{array}{l}\text { iPhone } 4 \text { and } \\
\text { iPad } 3\end{array}$ & $\begin{array}{l}8 \text { videos from various gen- } \\
\text { res with } 1280 x 720 \text { pixels res- } \\
\text { olution from DIEM project } \\
\text { (along with the eye-tracking } \\
\text { data). A total of } 80 \text { video } \\
\text { clips with various bit rates } \\
\text { for evaluation both on the } \\
\text { iPhone and iPad. }\end{array}$ & $\begin{array}{l}\text { Subjective assessment of region-of-interest } \\
\text { (ROI) based prioritization on mobile de- } \\
\text { vices across different content types, screen } \\
\text { resolutions, target bit rates and the two ROI } \\
\text { detection methods. }\end{array}$ \\
\hline$[44]$ & H.264/AVC & $\begin{array}{l}\text { Motorola } \\
\text { Atrix smart- } \\
\text { phone and } \\
\text { Motorola } \\
\text { Xoom tablet } \\
\end{array}$ & $\begin{array}{l}\text { LIVE mobile visual quality } \\
\text { assessment (VQA) database } \\
\text { consists of } 200 \text { distorted } \\
\text { videos and over } 50 \text { subjects. }\end{array}$ & $\begin{array}{l}\text { Subjective evaluation of visual quality with } \\
\text { fluctuations in video quality or distortions } \\
\text { on a long video sequences. Instantaneous } \\
\text { ratings as well as overall ratings are cap- } \\
\text { tured for each video. }\end{array}$ \\
\hline$[10]$ & H.264/AVC & $\begin{array}{l}\text { Google Nexus } \\
\text { One }\end{array}$ & $\begin{array}{l}28 \text { movie trailers from vari- } \\
\text { ous genres with } 2-3 \text { minutes } \\
\text { duration. }\end{array}$ & $\begin{array}{l}\text { Watching a streamed video in an every- } \\
\text { day life context (no constraint on when and } \\
\text { where they watch). Questions such as those } \\
\text { concerning video quality, playback, and con- } \\
\text { tent and user's physical context had to be } \\
\text { answered after the video playback. }\end{array}$ \\
\hline$[7]$ & H.264/AVC & $\begin{array}{l}\text { Google Nexus } \\
\text { S }\end{array}$ & $\begin{array}{l}50 \text { samples of video clips } \\
\text { with different qualities ob- } \\
\text { tained from three types of } \\
\text { video content. }\end{array}$ & $\begin{array}{l}\text { Development of temporal quality metric to } \\
\text { measure the quality of streaming videos. } \\
\text { The mean opinion score (MOS) was col- } \\
\text { lected from } 17 \text { subjects. }\end{array}$ \\
\hline$[46]$ & $\begin{array}{l}\text { No Infor- } \\
\text { mation } \\
\text { Available }\end{array}$ & $\begin{array}{l}\text { Simulated } \\
\text { feature phone } \\
\text { and smart- } \\
\text { phone screens }\end{array}$ & $\begin{array}{l}\text { Three types of video contents } \\
\text { including news, sports, and } \\
\text { music. A total of } 54 \text { video } \\
\text { conditions with various bit } \\
\text { rates, frame rates, and audio } \\
\text { quality with } 20 \text { seconds dura- } \\
\text { tions. }\end{array}$ & $\begin{array}{l}\text { Field experiments regarding the choice be- } \\
\text { tween video quantity and video quality } \\
\text { for } 25 \text { bandwidth-constrained low-income } \\
\text { users. }\end{array}$ \\
\hline$[52]$ & H.264/AVC & iPhone 3GS & $\begin{array}{l}30 \text { custom test clips encoded } \\
\text { with various quantization pa- } \\
\text { rameters, spatial resolutions, } \\
\text { and frame rates. }\end{array}$ & $\begin{array}{l}\text { Finding the lowest acceptable video quality } \\
\text { on mobile devices for videos with different } \\
\text { type of contents and durations. Analyzing } \\
\text { the impact of user's characteristics and pref- } \\
\text { erences and encoding quality. }\end{array}$ \\
\hline$[11]$ & $\begin{array}{l}\text { H.264 Scal- } \\
\text { able Video } \\
\text { Codec } \\
\text { (SVC) }\end{array}$ & $\begin{array}{l}\text { iPod classic } \\
\text { generation } 5.5\end{array}$ & $\begin{array}{l}48 \text { test clips are encoded at } 2 \\
\text { spatial resolutions, } 3 \text { tempo- } \\
\text { ral layers, and } 4 \text { quality lay- } \\
\text { ers. }\end{array}$ & $\begin{array}{l}\text { Subjective evaluation on the impact of } \\
\text { multi-dimensional scalability of SVC en- } \\
\text { coded video on human perceived quality for } \\
\text { low resolution videos. }\end{array}$ \\
\hline$[27]$ & H.264/AVC & Nokia 6630 & $\begin{array}{l}48 \text { video clips, each with a } \\
\text { duration of } 60 \text { seconds, with } \\
\text { news, sports, music, and car- } \\
\text { toon video content. }\end{array}$ & $\begin{array}{l}\text { Video quality experiments with } 30 \text { partici- } \\
\text { pants, comparing acceptability and satisfac- } \\
\text { tion of quality in three real usage contexts } \\
\text { (i.e., at the train station, bus, and cafe). }\end{array}$ \\
\hline$[49]$ & H.264/AVC & $\begin{array}{ll}\text { VPA } & \text { IV } \\
\text { UMTS/WLAN }\end{array}$ & $\begin{array}{l}\text { Two sets of } 5 \text { video se- } \\
\text { quences, each having a } 10 \\
\text { second duration and } 352 \times 288 \\
\text { pixel resolution. }\end{array}$ & $\begin{array}{l}\text { Estimation of perceived video quality for } \\
\text { mobile streaming. The MOS values were ob- } \\
\text { tained from } 36 \text { subjects, } 26 \text { people for the } \\
\text { training set and } 10 \text { people for the test set. }\end{array}$ \\
\hline
\end{tabular}

applications. The compressed domain offers an alternative approach for object detection and tracking with a faster processing time and fewer computational complexities $[63,14]$. Instead of using original pixel data, it uses encoded information, such as motion vectors or DCT coefficients. For example, a frame can be divided into several block-level fragments on the basis of the homogeneity of motion vectors or DCT coefficients; then, each fragment is merged into a similar neighboring frag- ment, and classified as foreground or background [63]. This approach is generally less accurate as its motion vectors are extracted from a bit stream that are sparsely assigned to blocks instead of pixels. Using encoded information, it is also hard to distinguish several objects in the presence of occlusions.

Many saliency techniques have been proposed in the literature and the review for such can be found in [5]. This work uses the spectral analysis model to obtain 


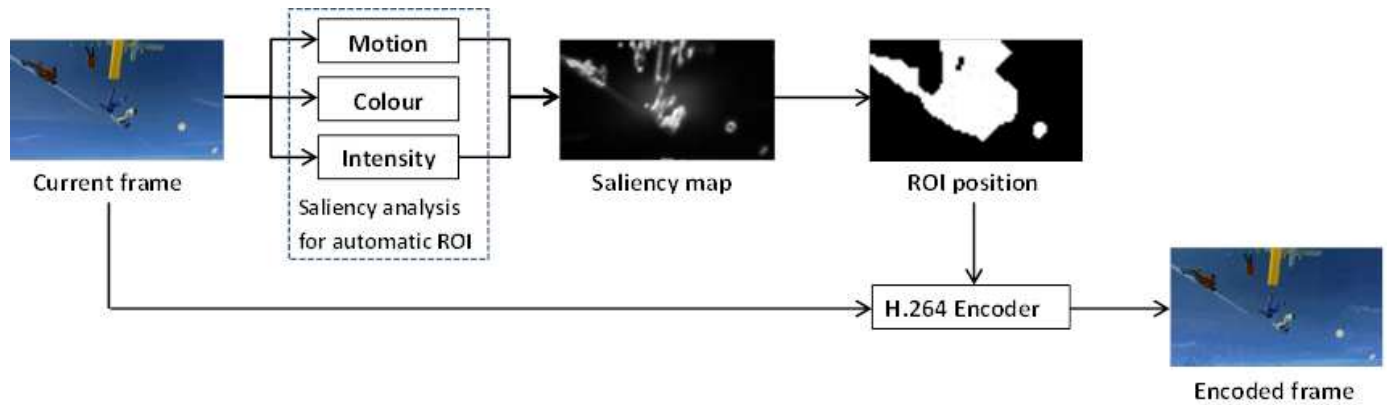

Fig. 1 The framework of ROI-based video encoding. The saliency map is obtained by analyzing motion, color, and intensity of a frame in a holistic manner. Assuming that the high salience in the saliency map is likely to attract attention, the ROI position map is generated from the top-valued positions. The H.264 encoder will assign lower quantization parameters for ROI and higher parameters for non-ROI.

a saliency map for automatic ROI detection. The spectral model is simple to implement while adhering to the saliency principle, and is also fast [5] because our aim is to achieve a real-time solution. The Fourier phase spectrum of an image contains crucial information about its geometry such as contours. The reconstruction of the phase spectrum of an image indicates the position of "objects" if there is less periodicity or less homogeneity in the vertical or horizontal orientation. This information has been exploited in $[3,16]$ to detect salient objects in the image. In addition, a top-down channel which searches for human faces has also been added to direct the automatic ROI detection since faces are often occurred in videos, and it was found in [6] that observers will fixate on a face within their first two fixations in a free-viewing task over $80 \%$ of the time.

\subsection{Region-of-Interest Coding Prioritization}

One form of ROI-based video encoding prioritization for the purpose of varying quality of ROI in the video is foveated video coding. Typically, it is used to reduce the bit rate and most of the reported studies show that it does not result in subjective quality degradation. The coding priority is achieved by giving maximum resolution to foveal regions, which are the fixation regions perceived by HVS, while at the same time removing redundant visual information in the periphery. The aim is to gain the highest possible compression with as little effect on perceived quality as possible $[51,58]$. Results show that this method does not degrade subjective quality while high compression gain can be achieved [45, 36]. Similar to the foveated coding approach, the ROIbased video encoding proposed in this paper prioritizes ROI by giving a higher bit allocation at the cost of less quality for the non- ROI regions. However, the aim is not to reduce the bit rate, but rather to improve the overall perceived quality of the video at the equal bit rate.

A great deal of work on ROI video coding employs facial detection $[8,62,28,39]$. While the presence of the face and human body is a cognitive-driven factor attracting visual attention, it is only applicable for videoconferencing applications and only recent studies such as $[54,4,37,17]$ have used the bottom-up saliency model to determine the ROI location. Therefore, the subjective quality of ROI video coding using automatic ROI has not been thoroughly evaluated in real scenarios, particularly in the mobile context. Some studies have indicated that using automatic ROI in ROI coding will not result in subjective quality degradation compared to using ROI derived from eye-tracking data $[15,45,34]$.

There are two different strategies when viewers judge image quality [33]. When judging a low quality image, the HVS attempts to look for image content in the presence of distortions. On the other hand, when judging a high quality image, the HVS look for distortions in the presence of the image. Hence, at lower bit rates, in which coding artefacts are clearly visible, it is expected that people tend to focus their attention on the interesting regions to comprehend the image content. Therefore, for the purpose of enhancing visual quality, allocating a higher quality for ROI will help to improve the overall perceived quality.

\subsection{Mobile Video Quality Assessment}

Video delivery on mobile devices poses many challenges, both technical and from the end-users requirements which affect the Quality of Experience (QoE), such as video coding capabilities, network conditions, human visual perception, and environments. Many researchers have conducted subjective video quality studies on mobile devices with various aims (selected examples are described in Table 1). Some investigations focus on study- 
ing the effect of video resolution, content, bit rate, and user characteristics (e.g., sex, age, frequent viewers), context, or all at the same time, for their acceptance of mobile video services $[27,11,52,46,10]$. Others aim to design objective algorithms that are capable of predicting visual quality against perceived quality with a high degree of correlation [49,7]. The lack of consistency in different studies compelled some researchers to create the LIVE Mobile VQA database which enables research into behavioral modeling of visual quality perception [44]. Some interesting findings from the study of this database suggest that the user seems to be more forgiving of poorer quality when viewing videos on a smaller screen and, at the same time, rewarding attempts to improve quality. Therefore, with the goal of developing efficient perceptually optimized video processing, the ROI coding approach can provide a balance between improving perceptual quality and the subjective QoE without increasing bit rate.

\section{Methods}

This section will describe the two adopted methods to obtain ROI which will be compared in our experimental studies, and the video encoding technique using ROI. The framework for ROI detection and coding is shown in Figure 1.

The screen resolution and size will have an impact on the perceptual quality $[42,30]$. With the fast development of smartphones and tablets (e.g., equipped with high resolution screen such as "retina displays"), it will be beneficial to conduct subjective studies using mobile devices that are relevant in todays world. Two types of mobile devices used in the experiments were the iPhone 4 and iPad $3^{\text {rd }}$ generation, both with the retina display technologies. The iPhone 4 has 960x640 pixels of resolution in a $7.5 \times 5.0 \mathrm{~cm}$ screen with 326 pixels per inch (ppi), and the iPad has 2048x1536 pixels resolution in a $19.7 \times 14.8 \mathrm{~cm}$ screen with 264 ppi. Comparative studies of ROI-based videos obtained by two different methods for determining the ROI will be made. The two methods for obtaining ROI are automatic ROI detection and eye-tracking based ROI.

\subsection{Automatic Region-of-Interest Detection}

The ROI is detected from a saliency map generated on a frame-by-frame basis. A video with a total number of $T$ frames is processed frame-by-frame as an image $I(x, y, t)$, where $x$ and $y$ are the location of each pixel and $t=1,2, \ldots, T$. Each frame is decomposed into luminance $Y$ and two chrominance components, $C_{r}$ and
$C_{b}$ [40]. The motion feature $M(t)$ is calculated by frame differencing, $M(t)=\|Y(t)-Y(t-\tau)\|$ to capture the temporal saliency between frames with the latency of $\tau$. The four features are represented by a quaternion image $q(t)$ which has four channels,

$q(t)=M(t)+C_{r}(t) \mu_{1}+C_{b}(t) \mu_{2}+Y(t) \mu_{3}$

where $\mu_{i}, i=1,2,3$, satisfies $\mu_{i}^{2}=-1, \mu_{1} \perp \mu_{2}, \mu_{2} \perp \mu_{3}$, $\mu_{1} \perp \mu_{3}, \mu_{3}=\mu_{1} \mu_{2}$. The $q(t)$ can be further represented in symplectic form, $q(t)=q_{1}(t)+q_{2}(t) \mu_{2}$, where $q_{1}(t)=$ $M(t)+C_{r}(t) \mu_{1}, q_{2}(t)=C_{b}(t)+Y(t) \mu_{1}$.

The frequency domain representation $Q(t)$ of quaternion image $q(t)$ is computed by means of Quaternion Fourier Transform (QFT) [50]. In polar form, $Q$ (t is dropped for clarity's sake) can be represented as $Q=$ $\|Q\| \exp ^{\mu \Phi}$, where $\Phi$ is the phase spectrum of $Q$ and $\mu$ is a unit pure quaternion. To obtain the image's phase spectrum $Q_{p}$, the $\|Q\|$ is set to unity (i.e., $\|Q\|=1$ ) or by computing $Q_{p}=\frac{Q}{\|Q\|}$. The spatio-temporal saliency map is obtained by convolving the $q_{p}$ with $2 \mathrm{D}$ smoothing filter $g$,

$s M(x, y, t)=g *\left\|q_{p}\right\|^{2}$

where $q_{p}$ is the inverse Fourier transform of $Q_{p}$.

Multi-scale analysis is performed by combining the saliency maps of different scales to highlight both local and global features [29]. To improve the results of ROI detection, the saliency map is augmented with maps created from high level knowledge of factors that are known to attract human attention. Hence, maps that locate faces using face detection and emphasize the center of the screen are used in combination with the saliency map to determine the ROI [20].

Each frame of an input video is divided into macroblocks (16x16 pixels in H.264/AVC format [59]) to generate the ROI, whereby a macroblock is defined as an ROI when the intensity of the map within the macroblock bounding box is above a certain (empirical) threshold. A smooth transition of ROI in consecutive frames is important to maintain perceived quality as salient objects on the screen rarely move in a sudden manner. This is achieved by setting upper and lower values so that the ROI region in a next frame will be changed only when the intensity of the map is not between these two values.

\subsection{Eye Tracking based Region-of-Interest}

Eye movements are generally influenced by low-level properties of stimuli (bottom-up cues such as color, intensity, and motion) and the idiosyncrasies of the viewer (top-down voluntary allocation of attention on relevant 


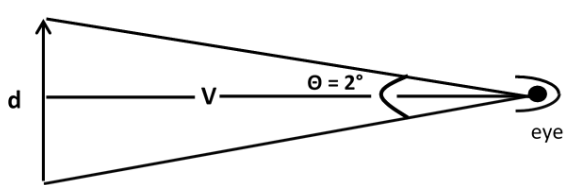

Fig. 2 Relationship between the viewing distance $V$ and the visual angle $\theta$.

features of the stimuli such as finding a face of a person) $[23,43,25]$. Given the same stimuli, eye movements should be consistent across viewers due to the bottomup factors. For example, [43] shows that motion is the strongest predictor of gaze location compared to other static cues.

For videos, Goldstein et al. found that eye movements from 20 subjects occupied less than $12 \%$ of the scene area in Hollywood movies for more than half of the viewing time [13]. A high degree of consistency where viewers were involuntarily drawn to the same location at the same time during dynamic scene viewing was also observed when viewers watched videos of real-world scenes $[41,43]$. The eye-tracking results that compared the original widescreen video and the postedited version at a smaller aspect ratio (using pans, cuts) found that the gaze position is consistent with the average distance over 18 clips less than $10 \%$ of the width of the original widescreen frame. These findings suggest that eye-tracking data will indicate the regions in videos that are important to the story and are reasonably consistent across viewers [24]. Due to the fact that the eye-tracking data on the mobile devices suitable for this study are not publicly available, the eyetracking ROI is derived from eye-tracking data collected and aggregated from participants watching videos on a 17 inch PC monitor, by the DIEM project ${ }^{1}$ [43]. This will provide a baseline comparison for automatic ROI.

The three main steps for obtaining the ROI from the eye fixation points are: (1) calculation of the high resolution region the human sees on both devices (i.e., iPhone and iPad); (2) calculation of the region size on test videos in terms of macroblocks, when these are viewed on actual devices; (3) Forming the ROI by enclosing each fixated point with a square of macroblocks the approximate size of high resolution region of an eyes retina. The details for these procedures are described as follows.

\subsubsection{Calculation of High Resolution Region}

Mobile devices are typically operated at an arm's length. Given that visual acuity in human vision extends to a 2 degree visual angle at arm's length, this means that

\footnotetext{
1 available at http://thediemproject.wordpress.com
}

people will only see a high resolution images within a few centimeters of the area surrounding the point of fixation. With the assumption of 2 degrees of visual angle [47], the region which people see in high details around the fixated points equals to $d=\tan (\theta) \times V$, where $d$ is the region height, $\theta$ is the visual angle, and $V$ is the viewing distance that is ideally measured from the fixated location on the screen to the eyes of viewer. The relationship between the viewing distance and the visual angle is depicted in Figure 2.

Using the formula, where the viewing distance is assumed to be $35 \mathrm{~cm}$ for the iPhone, this will correspond to an approximately $1.22 \mathrm{~cm} \times 1.22 \mathrm{~cm}$ region. In practice, however, people may operate different mobile devices with different viewing condition depending on the screen size, but still within an arm's length distance. Devices with a larger screen may likely be viewed from a further distance than devices with a smaller screen size. Thus, $50 \mathrm{~cm}$ of viewing distance is assumed for the iPad. This will correspond to an approximately $1.75 \mathrm{~cm} \times 1.75 \mathrm{~cm}$ region.

\subsubsection{Calculation of Actual Region Size in Devices}

Given that the pixel density of an iPhone screen is 326 ppi (pixels per inch), the region of visual acuity in terms of macroblocks can be calculated. Note that the video resolution used in this experiment is $1280 \mathrm{x} 720$ pixels, which correspond to $80 \times 45$ macroblocks. Due to the mismatch of the aspect ratio between the iPhone's native resolution and the videos resolution, the video is displayed on the iPhone with a resolution of $960 \times 540$ pixels. Hence, a macroblock on the iPhone is $12 \mathrm{x} 12$ pixels wide. Given a visual acuity of $1.22 \mathrm{~cm}$ which is equal to 156.86 pixels wide on the iPhone, it corresponds to approximately $13 \times 13$ macroblocks on test videos.

When the test videos are displayed on the iPad, the device attempts to fit the videos to the full screen size. However, the videos only occupy 2048x1152 pixel region and a macroblock on the iPad is displayed at 25.6x25.6 pixels wide. Given the visual acuity of $1.75 \mathrm{~cm}$ which is equal to 181.37 pixels wide and the pixel density of 264 ppi for the iPad, this corresponds to approximately $7 \times 7$ macroblocks on test videos.

\subsubsection{Formation of ROI based on Fixation Points}

Two regions of interest are created for both the iPhone and iPad which follow similar steps. First, the binary image is created with a resolution of $80 \times 45$; thus, every macroblock will correspond to one pixel in this image. For each video frame, macroblocks where eye fixation fell are represented as an active pixel (i.e., a binary one). 
Table 2 The eight videos from DIEM project used in the experiments.

\begin{tabular}{|c|c|c|c|}
\hline Video Name & Length & No. Subjects & Descriptions \\
\hline adrenaline_rush (AR) & $2: 11$ & 123 & $\begin{array}{l}\text { Shot of people parachuting with a blue sky in the background. The video } \\
\text { begins with a shot of an aircraft and four people parachuting. After three } \\
\text { seconds, the people parachuting are centred in the middle of screen. The } \\
\text { camera is then tracking a person with a blue parachuting suit. }\end{array}$ \\
\hline bullet_witch (BW) & $2: 28$ & 123 & $\begin{array}{l}\text { A video game trailer showing computer graphic animation. Most of the } \\
\text { scenes contain close-up shots of a female or male character with their } \\
\text { face and shoulder in various poses (sometimes holding a weapon). In } \\
\text { other scenes, male and female characters are shown in a standing pose } \\
\text { together. }\end{array}$ \\
\hline coral_reef_adventure (CR) & $1: 58$ & 42 & $\begin{array}{l}\text { The scene begins with a boat cruising at sunset. After two seconds, it } \\
\text { shows people working with computers at desks inside a workshop where } \\
\text { a boat, glass windows, and large hanging lamps are shown in the back- } \\
\text { ground. The last five seconds of the video shows people diving underwater } \\
\text { in coral reefs with colorful fish swimming around. }\end{array}$ \\
\hline dolphins (DP) & $2: 07$ & 124 & $\begin{array}{l}\text { The scenes contain sea cliffs covered with green vegetation and ocean } \\
\text { waves under a blue sky and clouds. The shot then slowly moves toward } \\
\text { the horizon away from the cliffs where the ocean and blue sky dominate } \\
\text { the scene. }\end{array}$ \\
\hline advert_bravia (AD) & $1: 26$ & 42 & $\begin{array}{l}\text { Sony Bravia advertisement. The scene begins with blue paint being dis- } \\
\text { charged from a hose in the corner of a building. This is followed by a } \\
\text { bird's eye view of multiple apartment buildings of a light brown color } \\
\text { and the surrounding parks. In the last three seconds, the scene shows } \\
\text { paint being fired upward in front of a building in successive waves. }\end{array}$ \\
\hline barcelona_extreme (BE) & $1: 08$ & 46 & $\begin{array}{l}\text { The scenes contain someone water skiing while pulled by a motorboat in a } \\
\text { river with concrete fences in the background. Shots of spectators watching } \\
\text { the sport (standing or in a boat) are sometimes shown throughout the } \\
\text { video. }\end{array}$ \\
\hline discoverers (DC) & $3: 02$ & 42 & $\begin{array}{l}\text { Shot of a large telescope moving in rotation inside an observatory that } \\
\text { lasts about half of the video length. This is followed by scenes of people } \\
\text { walking inside a building with bright lighting to contrast with the dark } \\
\text { background. }\end{array}$ \\
\hline mystery_nile (MN) & $1: 44$ & 42 & $\begin{array}{l}\text { Shot of someone water rafting in a rough river with a hill and blue sky } \\
\text { in the background. Scenes of someone standing on the hill are sometimes } \\
\text { shown throughout the video. The last two seconds of the clip shows the } \\
\text { tip of a yellow raft floating in the river with the hill and blue sky in the } \\
\text { background. }\end{array}$ \\
\hline
\end{tabular}
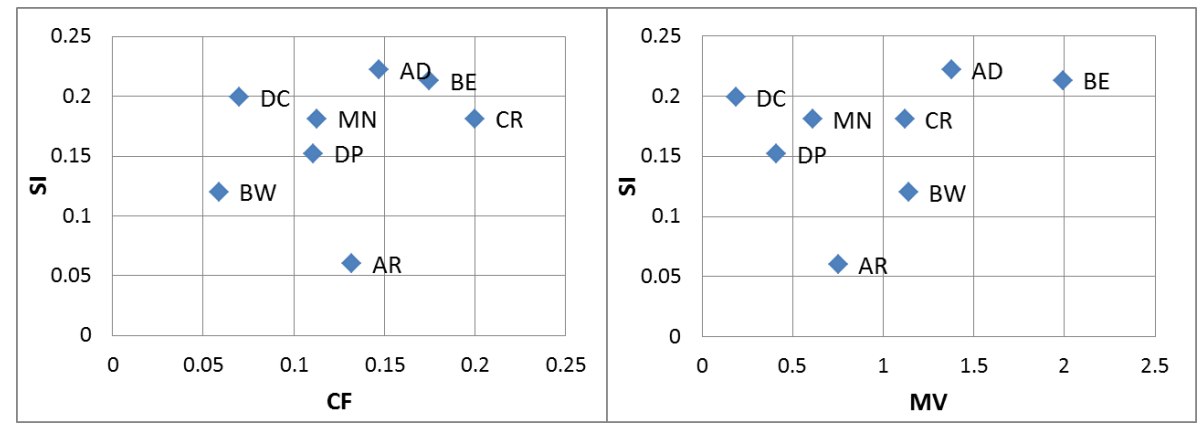

Fig. 3 Spatial information (SI) vs. colorfulness $(\mathrm{CF})$ and motion vectors (MV), respectively.

The final pixels value represents the amount of eye fixation points that fall into this particular macroblock after all points have been accumulated. The image is then convolved with a square with $13 \times 13$ of ones to acquire the ROI for the iPhone; and similarly, a square with $7 \times 7$ of ones to acquire the ROI for the iPad. However, with a large number of subjects in the eye-tracking data, this will generate a large ROI which can occupy the whole frame since the same subject will not always gaze to the point of interest that most would look toward in every frame. To obtain a more sensible ROI from the eye-tracking data, any ROI that is isolated from the majority of gaze locations is removed and then focus 
is set on the ROI where the majority of people would look. This is how the final ROI is obtained:

1. After the convolution, the thresholding operation is applied on the ROI image so that only pixel values greater than one are retained. This will remove any regions which are isolated (i.e., located a significant distance from the main ROI position) that are likely to be generated from only one subject's fixation points. At the same time, it will shrink the outer layer of the main ROI region. This can be tolerated since the extended area of the main ROI is chiefly caused by the large number of fixation points that sporadically fall within the vicinity of the object of interest.

2. Any isolated ROI that is less than $11 \times 11$ is removed for the final ROI binary image for the iPhone and any ROI that is less than $5 \times 5$ is removed for the iPad, respectively. Thus, any isolated ROI can only be retained if two or more subject's fixation points are located in close proximity. The ROI in the original video resolution is obtained by replacing each pixel in the ROI image with $16 \times 16$ pixels of zeros if the pixel's value is zero and $16 \times 16$ pixels of ones if the pixel's value is not zero.

\subsection{Encoding Video using ROI}

The ROI-based video encoding is implemented using a custom-developed x264 encoder, a state-of-the-art library which produces video bit stream in H.264/AVC format, (available at http://www.videolan.org, version 0.88 as of 2010) and capable of allocating different amount of bits to the ROI/non-ROI region by changing the quantization parameter (QP) values of macroblocks. The regions of interest are pointed out to the encoder as macroblock positions within a frame. The ROI-based encoding videos were created using the two-pass encoding process to obtain a more accurate target bit rate with different qualities in ROI and non-ROI regions. The quality of the non-ROI is reduced by increasing the value of the quantization parameter to six higher than the QP values which are assigned by the encoder during the first pass. The QP-6 was decided based on empirical experiments to ensure that non-ROI quality is lower than the ROI (i.e., if the QP was set too high, the non-ROI quality would be too blocky; if the QP was set too low, there would be no obvious bit rate saving). This ensures that PSNR and SSIM measured higher in the ROI over the non-ROI. Not all macroblocks in a frame have the same QPs. The QPs of macroblocks were determined using the constant bit rate (CBR).
The turbo mode on the first pass in x264 was selected with encoding settings such as the sub-pixel motion estimation. The mode decision was set to the sum of absolute transformed differences (SATD) mode decision and the number of reference frames used was one. The adaptive spatial transform size was disabled and the integer pixel motion estimation method was set to diamond search with a radius of one. No B-frames were used because the older version of the iOS video player used did not offer such support. The maximum GOP size was set to 50 to allow seeking for videos with a higher precision, and the other x264 parameters were set to the default.

The normal quality video was encoded with an average target bit rate encoding mode. The same target bit rate was imposed upon the ROI-based video. Akamai [1] suggested $250 \mathrm{kbps}$ for encoding a low quality video for streaming over a $3 \mathrm{G}$ network. In this paper, a target bit rate starts at $300 \mathrm{kpbs}$ for a low quality video and increases gradually up to $1100 \mathrm{kbps}$ for a high quality video.

Target bit rates of $300 \mathrm{kbps}, 400 \mathrm{kbps}, 700 \mathrm{kbps}$, and $1100 \mathrm{kbps}$ were prepared for each video clip using the automatic ROI. Only two target bit rates (300 kbps and $700 \mathrm{kbps}$ ) were selected for encoding videos using the eye-tracking based ROI, thus reducing the number of test clips for shorter subjective testing duration to avoid visual fatigue. The two bit rates also represent low and medium quality videos. Since it is difficult to aim for the exact specified target bit rate, a $\pm 3 \mathrm{kbps}$ deviation from the target was allowed.

\section{Experimental Setting}

This section presents and analyzes the testing materials used in the user study and describes procedures for subjective testing.

\subsection{Testing Material}

Eight representative videos were selected to cover a variety of content characteristics from the DIEM project database for our experiments. The DIEM database contains mostly TV video content such as advertisements, documentaries, film trailers, and music videos to collect eye tracking data from people when they watch these videos. The eight videos were cut to 12 seconds in length and had a resolution of $1280 \times 720$ pixels at frame rates of 30 seconds with no audio. The short video durations (i.e., 10 and 15 seconds) have been used for mobile video quality assessments to obtain statistically meaningful 
results $[48,44]$. The video name and the number of individuals watching the video in which the eye-tracking data were collected, as well as descriptions, are shown in Table 2. The 12-second video segments were selected based on manual observations to best match the video content descriptions.

For each type of video content, the original clip was encoded with four target bit rates using automatic ROI coding and normal coding as a baseline, and two target bit rates using eye tracking based ROI. Thus, a total of 80 test clips were created for each device.

The content characteristics of test videos are quantitatively analyzed along with the dimensions of color, space, and time by computing the spatial information (SI) [2], colorfulness (CF) [19], and motion vectors (MV) so that comparisons between test clips can be visualised [60]. The SI and CF values are averaged over all frames for each test clip.

The raw SI, CF, and MV values for each clip are shown in Figure 3. From the plots, the distribution of clips show significant content differences along the dimensions of color, space (texture), and time (motion). In terms of colorfulness, test videos are distributed evenly with video $\mathrm{CR}$ having the highest value and video BW having the lowest value. In addition, test videos are distributed evenly in terms of motion with video BE having the highest motion due to it displaying fast moving objects and containing many scene cuts. On the other hand, video DC has the slowest motion content as it contains slow moving scenery. Most videos have medium to rich textures, except for video AR.

\subsection{Subjective Testing Procedures}

The subjective viewing test to measure the impact of ROI video coding was conducted using the two mobile devices: the iPhone 4 and the iPad $3^{r d}$ generation, both with the retina display technologies and touchscreen interfaces. A customized mobile app was developed to play videos and to collect participants responses. A written instruction sheet summarising the test procedures was given to each subject at the start of every study. The motivation and instructions of the study were explained to every participant.

The absolute category rating (ACR) was used for assessing the video quality [59]. The test sequences were presented one at a time to the viewer, and were rated independently using a continuous quality scale. An onscreen slider was made to appear by tapping the screen twice so that the viewers could cast their judgments. The test clips were put into four groups to minimize visual fatigue rather than displaying all clips in a sequence at once. The test clips were randomly positioned

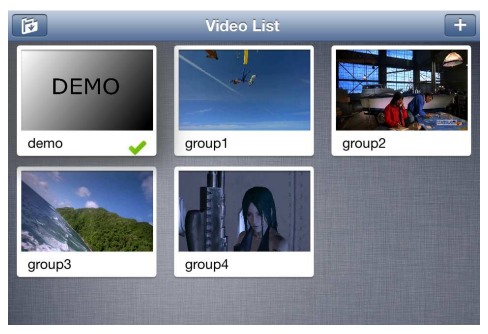

(a) Home Screen

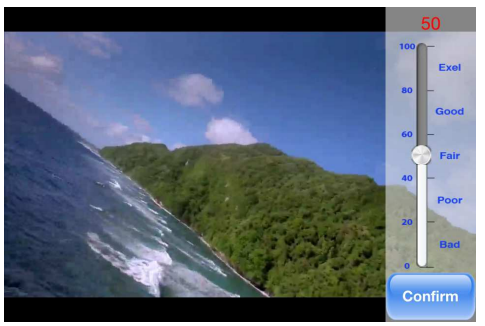

(b) Rating Slider

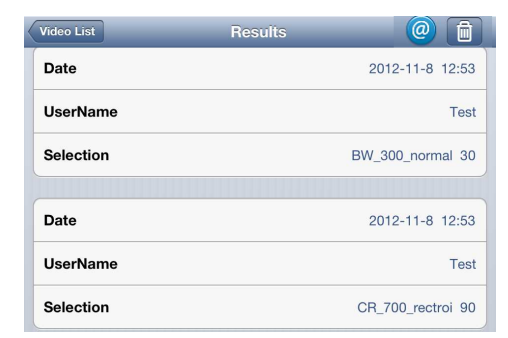

(c) Recorded Responses

Fig. 4 Screenshots of customized iPhone 4 application for the subjective viewing test.

in four groups. Once all clips in one of the group were rated, the application showed the home screen (see Figure 4.a.) so that the subject could continue to the next group by tapping the group icon.

The user studies were conducted in two separate sessions because of the large number of video clips used in this study. The first session used the four test videos $\mathrm{AR}, \mathrm{BW}, \mathrm{CR}$, and DP, and the second session used the remaining test videos $\mathrm{AD}, \mathrm{BE}, \mathrm{DC}$, and $\mathrm{MN}$. The contents of videos used in the second user study have rich textures, with video $\mathrm{BE}$ having the highest motion along with video AD. For each user study session, one iPhone and one iPad were used. Participants watched a total of 40 video clips on the iPhone and 40 clips on the iPad, and cast their judgments.

The user study started with a five minutes briefing. During the briefing, the training videos (the demo group) were presented with instructions on how the assessment would be conducted and answered any questions that arose until the subjects felt comfortable undertaking the rest of experiments by themselves. The experiment was conducted indoors in an office with fluorescent lighting. The subject was instructed to handle 
Table 3 The percentage (\%) of fixation points that fell within the automatic ROI for both devices, averaged over the number of subjects and frames.

\begin{tabular}{|c|c|c|c|c|c|c|c|c|}
\hline Device & $A R$ & $B W$ & $C R$ & $D P$ & $A D$ & $B E$ & $D C$ & $M N$ \\
\hline iPhone & 92.5 & 85.9 & 81.7 & 81.1 & 49.0 & 79.1 & 82.1 & 70.7 \\
iPad & 79.8 & 63.4 & 51.5 & 53.6 & 28.8 & 53.6 & 58.4 & 65.5 \\
\hline
\end{tabular}

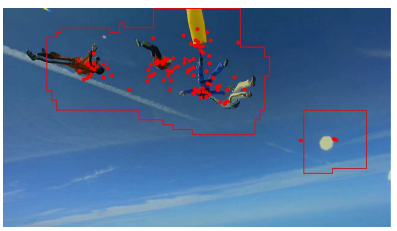

(a) Video AR

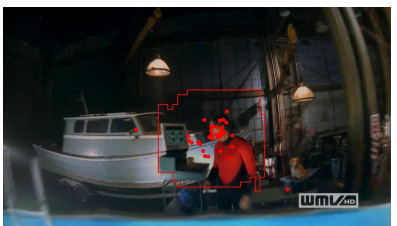

(c) Video CR

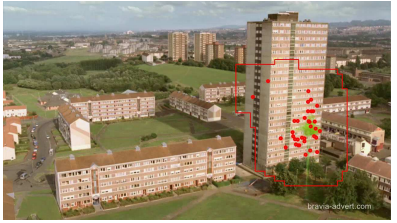

(e) Video AD

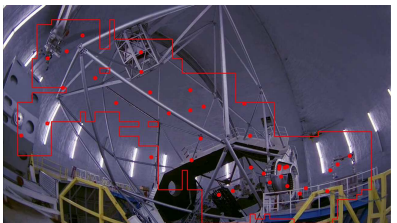

(g) Video DC
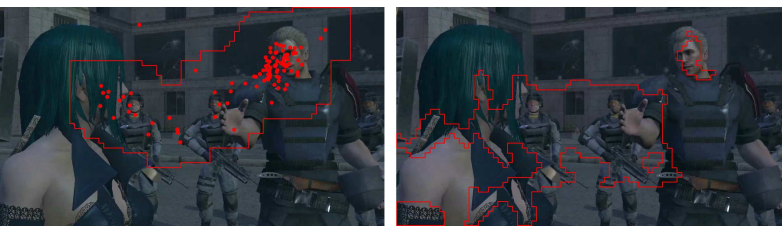

(b) Video BW
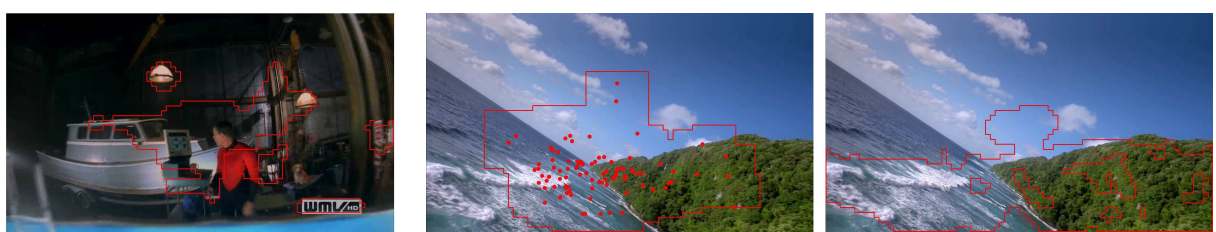

(d) Video DP
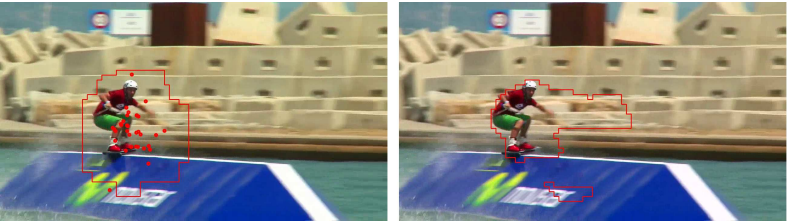

(f) Video BE
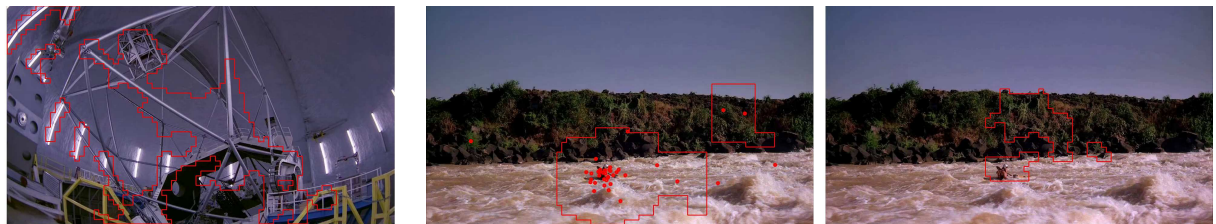

(h) Video MN

Fig. 5 The eye-tracking based ROI (enclosed by the red lines) which is determined from the eye fixation locations (red circles) is shown together with the automatic ROI (enclosed by the red lines) from a frame in videos AR, BW, CR, DP, AD, BE, DC, and MN respectively. The average area of automatic ROI in a particular video is constrained to have an approximately similar size with the eye-tracking based ROI.

and view the devices in a way that felt comfortable without forcing the distance between the user and the device. The examiner was present at the beginning of the user study to make sure the subjects knew how to operate the devices themselves. A verbal confirmation of normal color vision was obtained from the subject. The outlines of the subjective video quality assessment are as follows:

1. The subject starts the assessment with clips in the first group by tapping the group 1 icon on the home screen.

2. The subject is asked to rate every video in the first group. Each video is playing on a loop and when the subject has made a decision, they simply tap the screen twice to rate the video they are cur- rently watching (see Figure 4.b.). This can be accomplished while the video is still playing. The onscreen slider MOS with a continuous scale and an integer scale from 0 ("Bad") to 100 ("Excellent") is used to judge the quality. The user can take as much time as they need to rate the clip.

3. Once a video clip is rated, a three-second mid-grey mask appears on the screen before the next video clip is displayed. The assessment is continued until all videos are rated in a group.

4. The home screen will be shown after all clips in one group have been rated. The subject simply moves to the next group by tapping the group icon.

The subjective studies included a total of 54 participants (34 male and 20 female) who were students 
Table 4 The average $F_{1}$ measure of automatic ROI against the eye-tracking based ROI for the two devices.

\begin{tabular}{|c|c|c|c|c|c|c|c|c|}
\hline Device & $A R$ & $B W$ & $C R$ & $D P$ & $A D$ & $B E$ & $D C$ & $M N$ \\
\hline iPhone & 0.64 & 0.59 & 0.56 & 0.62 & 0.35 & 0.54 & 0.61 & 0.43 \\
iPad & 0.58 & 0.45 & 0.36 & 0.45 & 0.21 & 0.43 & 0.49 & 0.37 \\
\hline
\end{tabular}

from a university. The subjects did not work in the field of video quality assessment. A movie voucher was offered to each participant as a reward. For each user study session, the participant took approximately 3545 minutes to complete the tasks, which included five minutes of briefing. In the first session, a total of 25 assessments from 31 participants were collected, 19 participants watched both on the iPhone and iPad, while 6 participants watched only on the iPhone and another 6 watched only on the iPad. In the second session, a total of 20 assessments from 23 participants were collected, 17 participants watched both on the iPhone and iPad, while 3 participants watched only on the iPhone and another 3 watched only on the iPad. Evaluation responses for data analysis were recorded on devices directly (see Figure 4.c.).

\section{Results}

The screenshots of videos using automatic ROI and eyetracking based ROI used in the user study experiments are shown in Figure 5. The eye-tracking based ROI is shown along with the eye fixation points.

\subsection{ROI Detection Accuracy}

The ROI detection accuracy is measured using the ground truth, which is the eye-tracking data. The eye-tracking data contains information about the fixation locations of subjects for each video frame. Judd et al. suggest that 39 viewers provides fairly accurate ground truth fixation map due to the ability of the fixation map to predict new fixations under the receiver operating characteristics (ROC) curve in their eye-tracking study using 300 natural images [26]. The eye-tracking data used in this study were collected from more than 40 subjects.

The percentage of fixation points that fell within the automatic ROI for both the iPhone and iPad, averaged over the number of subjects and frames, is shown in Table 3. Overall, the percentage of fixation points that fell within the ROI region is greater on the iPhone compared to the iPad. This is because the automatic ROI has a larger area in terms of percentage of the total frames size on the iPhone due to the iPhone's smaller screen size and resolution when both devices are operated at an arms length (calculation in Section
3.2.2 shows the width of the ROI region on test videos is about twice as much in terms of macroblocks when viewed on the iPhones screen compared to the iPad). This suggests that a more accurate ROI detection is needed for ROI-based videos viewed on a larger screen (e.g., iPad) compared to a smaller screen (e.g., iPhone), when they are operated with a similar viewing distance for both devices.

Among the test video clips, video AR has a higher accuracy of automatic ROI detection in which ROI covers $92.5 \%$ of fixation points on the iPhone. Video AR in particular contains people parachuting from an airplane in the uniform-textured background (i.e., sky), and these stand-out objects are easier to detect using ROI detection algorithm. It is harder for the algorithm to recognize objects that are considered salient in the rich textured background (i.e., contain high spatial information such as video $\mathrm{AD}$ ).

Not all fixations which fall within the ROI will be detected by the automatic tool. In this study, a fixation which falls within the ROI but is not detected by the automatic tool is analyzed by calculating the similarity between automatic ROI and eye-tracking based ROI using the $F_{1}$ metric which combines precision and recall values. Since the region of interest is defined in terms of macroblocks, the $F_{1}$ metric is given as

$F_{1}=2 \frac{\text { precision } \times \text { recall }}{\text { precision }+ \text { recall }}$

where precision gives the fraction of macroblocks from the eye-tracking based ROI that are relevant to the estimated ROI and the recall gives the fraction of macroblocks that are successfully estimated with respect to the eye-tracking based ROI. The precision and recall are defined as

precision $=\frac{n_{g e}}{N_{e}}$, recall $=\frac{n_{g e}}{N_{g}}$

respectively, where,

$n_{g e}$ : total number of macroblocks in estimated ROI that are from eye-tracking based ROI.

$N_{e}$ : total number of macroblocks in the estimated ROI. $N_{g}$ : total number of macroblocks in the eye tracking based ROI.

Note that in this experiment, the value of $N_{e}$ and $N_{g}$ are similar because automatic ROI and eye tracking based ROI are constrained to have the same size. The 
Table 5 The t-value and p-value from a paired-sample t-test to examine whether the ROI-based videos scores are significantly higher than normal videos on the iPhone and iPad.

\begin{tabular}{|c|c|c|c|c|}
\hline & \multicolumn{2}{|c|}{ iPhone } & \multicolumn{2}{c|}{ iPad } \\
\hline bit rate $(\mathrm{kbps})$ & t-value & $\mathrm{p}$-value & $\mathrm{t}$-value & $\mathrm{p}$-value \\
\hline 300 & $t_{175}=-4.92$ & $p<0.001$ & $t_{173}=-5.48$ & $p<0.001$ \\
400 & $t_{172}=-3.41$ & $p=0.001$ & $t_{175}=-5.86$ & $p<0.001$ \\
700 & $t_{178}=-3.89$ & $p<0.001$ & $t_{169}=-2.46$ & $p=0.015$ \\
1100 & $t_{176}=-1.10$ & $p=0.271$ & $t_{173}=-5.84$ & $p<0.001$ \\
\hline
\end{tabular}
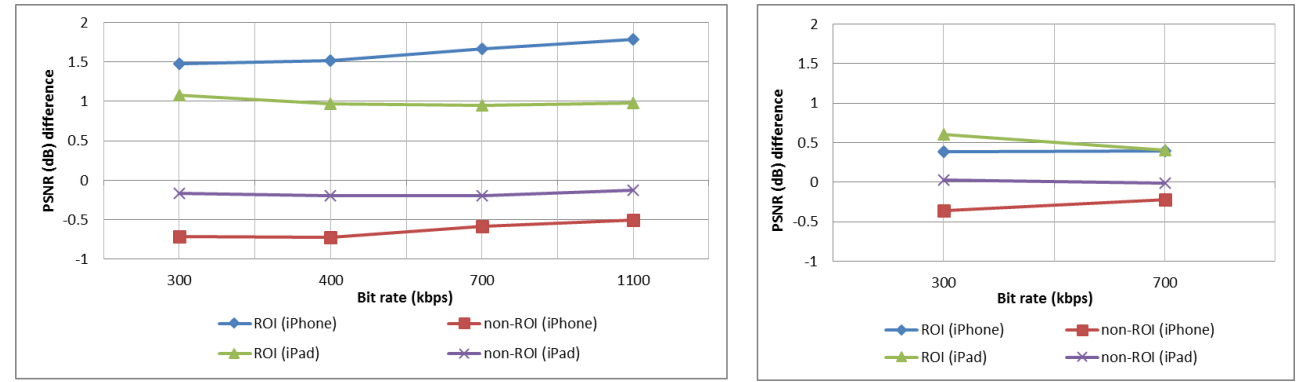

Fig. 6 The PSNR difference between ROI-based coding and normal coding videos, averaged for eight test videos, within the ROI and non-ROI on the iPhone and iPad. The ROI is determined using automatic ROI (left figure) and using eye tracking based ROI (right figure).
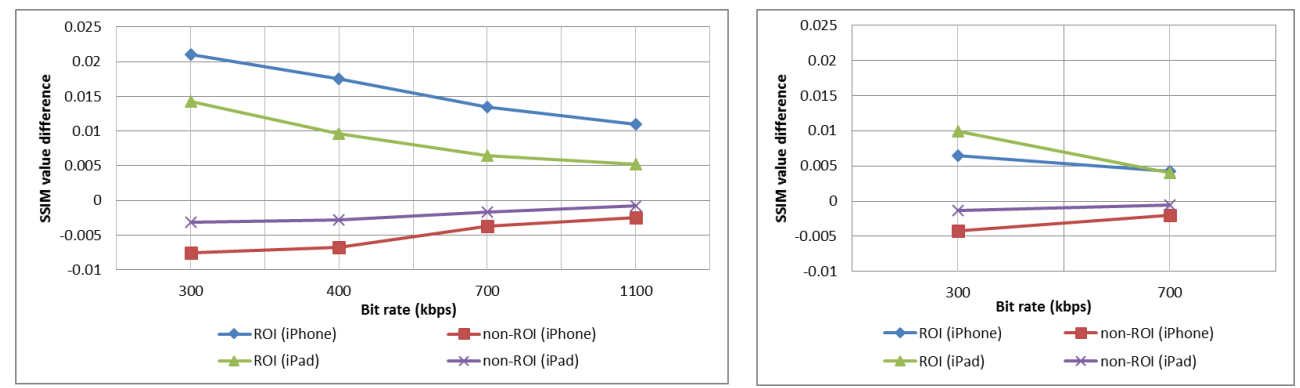

Fig. 7 The SSIM difference between ROI-based coding and normal coding videos, averaged for eight test videos, within the ROI and non-ROI on the iPhone and iPad. The ROI is determined using automatic ROI (left figure) and using eye tracking based ROI (right figure).

$F_{1}$ score of 1 means that the estimated ROI overlaps perfectly with the eye-tracking based ROI.

On the iPhone, Table 4 shows that the automatic ROI covers more than $50 \%$ of the eye tracking based ROI where, for most videos, more than $80 \%$ of the fixation points fell within the automatic ROI, except for videos $\mathrm{AD}$ and $\mathrm{MN}\left(F_{1}=0.35\right.$ and $F_{1}=0.43$, respectively). For the iPad however, the automatic ROI covers less than $50 \%$ of the eye-tracking based ROI, except for video $\mathrm{AR}\left(F_{1}=0.58\right)$. One of the reasons is that automatic ROI has a larger area in terms of percentage of the total frame's area on the iPhone compared to the iPad. This is reflected by the large percentage of fixation points that fell within the automatic ROI as shown in Table 3 . On the iPad, the lower $F_{1}$ score is caused by fewer fixation points falling within the automatic ROI.

Any fixation which falls within the eye-tracking based ROI but not in automatic ROI will determine the fixa- tions which fall within the ROI but are not detected by the automatic tool. For example, video AR achieves an $F_{1}$ score of 0.64 and ROI covers $92.5 \%$ of the fixation points on the iPhone. This means $7.5 \%$ of the fixation points are not detected by the automatic tool and fall within the $36 \%$ of the eye-tracking based ROI outside the automatic ROI.

5.2 Objective Assessment on Quality Improvement of ROI-based Videos

Two objective quality measurements, PSNR and SSIM index [57], are used to confirm that the ROI has higher quality compared to other regions. Figure 6 shows PSNR differences between ROI-based coding videos (using automatic ROI) and normal coding videos, averaged for eight test videos, within the ROI and non-ROI on the iPhone and iPad. The graph on right in the same fig- 

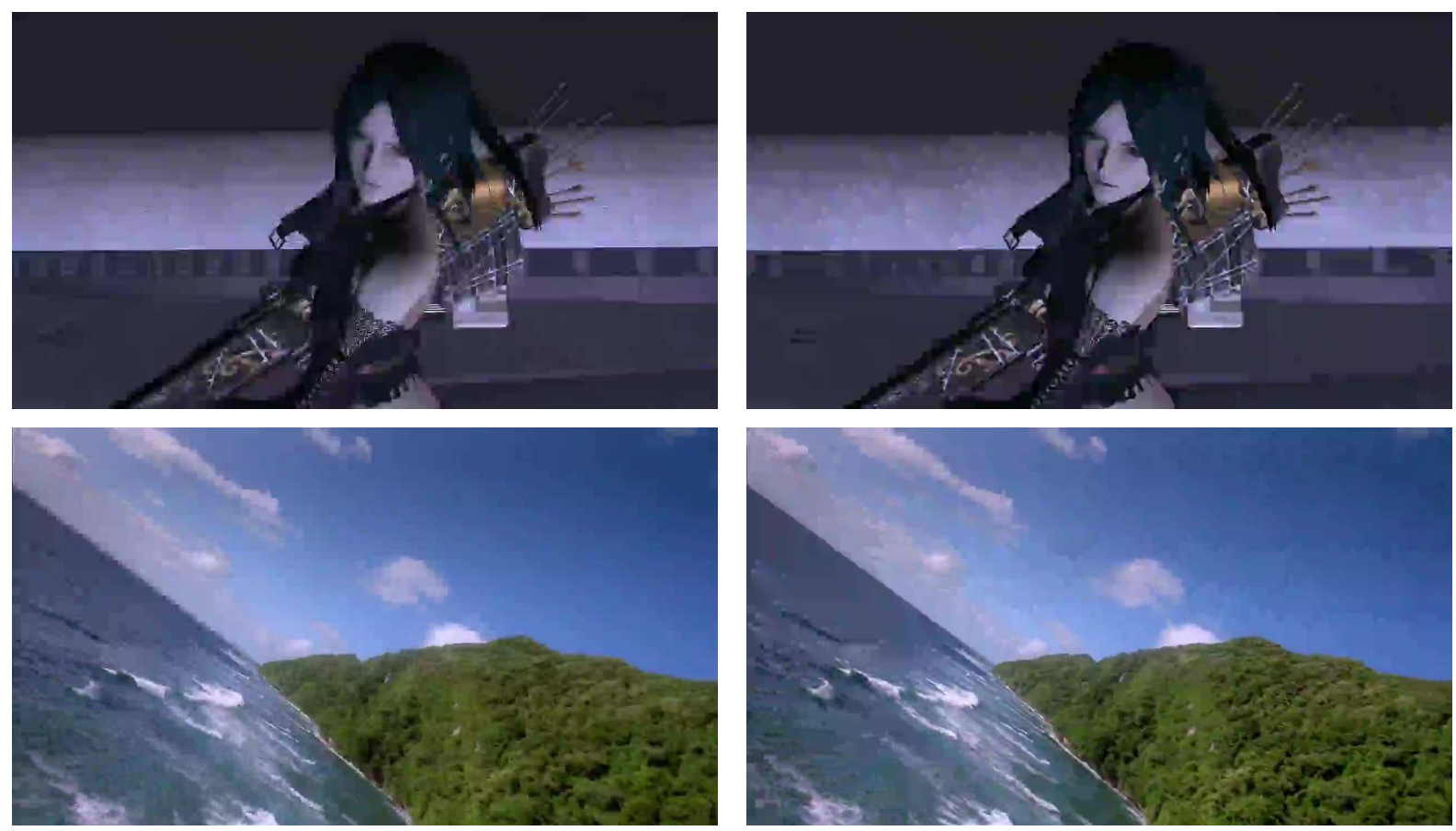

Fig. 8 Screenshots from normal (left) and ROI encoded (right) videos at 300 kbps for video BW (top) and DP (bottom).

Table 6 The t-value and p-value from a paired-sample t-test to examine whether the ROI-based videos scores using automatic ROI are significantly higher than the scores of ROIbased videos using eye tracking based ROI.

\begin{tabular}{|c|c|c|c|c|}
\hline & \multicolumn{2}{|c|}{$300 k b p s$} & \multicolumn{2}{c|}{$700 \mathrm{kbps}$} \\
\hline & t-value & $\mathrm{p}$-value & t-value & $\mathrm{p}$-value \\
\hline iPhone & $t_{175}=-0.67$ & $p=0.49$ & $t_{178}=1.29$ & $p=0.19$ \\
iPad & $t_{173}=0.88$ & $p=0.39$ & $t_{169}=1.97$ & $p=0.05$ \\
\hline
\end{tabular}

ure shows the PSNR difference between ROI-based coding videos (using eye-tracking based ROI) and normal coding videos, averaged for eight test videos, within the ROI and non-ROI on both devices. Note the positive PSNR differences in the ROI (suggesting a better quality ROI of ROI-based videos compared to normal videos). Figure 7 shows differences between ROI-based coding videos and normal coding videos within ROI and non-ROI that are calculated using an SSIM index. Figure 8 shows the snapshots from normal and ROIencoded videos at $300 \mathrm{kbps}$ for viewing on the iPhone.

\subsection{Subjective Testing Results}

The combined responses from the two sessions of user studies resulted in 2160 data points. The 41 data points which were the outliers were identified by plotting the box plot for each video content and bit rate category, and subsequently removed from the analysis.

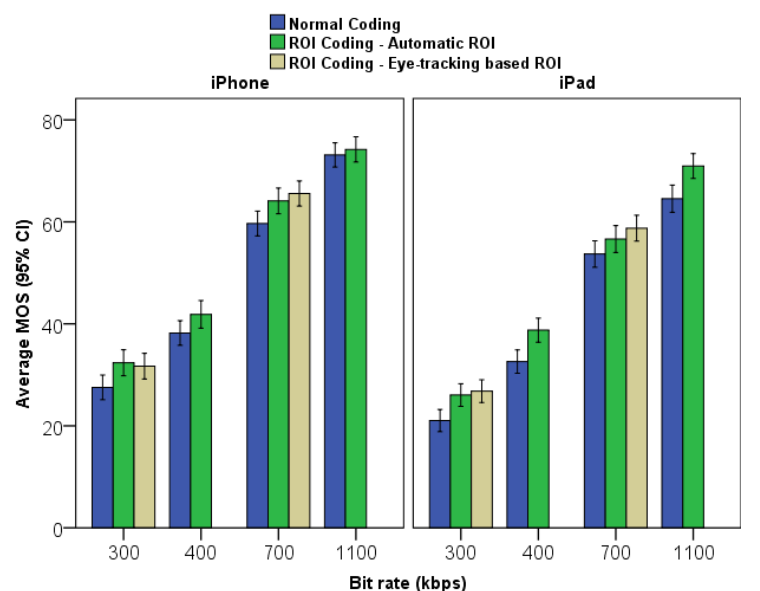

Fig. 9 The mean opinion score averaged for the eight videos for iPhone (left) and iPad (right) across different target bit rates for ROI-based videos with automatic ROI, ROIbased videos with eye-tracking based ROI, and normal coding videos.

The MOS averaged for the eight videos across different bit rates are shown in Figure 9, where the span of each vertical line indicates a $95 \%$ confidence interval. It was found that the ROI-based coding videos have better perceptual quality when they are viewed both on the iPhone and iPad. A set of paired-sample t-tests was conducted to examine whether the ROI-based videos were effective in improving perceived quality over normal videos. According to the null hypothesis, the scores of both coding strategies come from the distributions 
Table 7 Difference mean opinion score (DMOS) between the scores of ROI based coding and normal coding averaged across different contents and bit rates for test videos classified in three classes (i.e., high, medium, or low) based on the amount of spatial information (SI), colorfulness (CF), and motion vectors (MV).

\begin{tabular}{|l|l|l|l|}
\hline Metric & Value & Video & DMOS \\
\hline \multirow{4}{*}{ SI } & High & $\begin{array}{l}\text { AD, BE, DC, CR, } \\
\text { MN }\end{array}$ & 1.9 \\
\cline { 2 - 4 } & Medium & DP, BW & 3.8 \\
\cline { 2 - 4 } & Low & AR & $\mathbf{8 . 6}$ \\
\hline \multirow{3}{*}{ CF } & High & CR, BE & 3.4 \\
\cline { 2 - 4 } & Medium & AD, AR, MN, DP & $\mathbf{4 . 1}$ \\
\cline { 2 - 4 } & Low & DC, BW & 1.3 \\
\hline \multirow{3}{*}{ MV } & High & BE, AD & 1.9 \\
\cline { 2 - 4 } & Medium & BW, CR, AR, MN & $\mathbf{4 . 4}$ \\
\cline { 2 - 4 } & Low & DP, DC & 2.2 \\
\hline
\end{tabular}

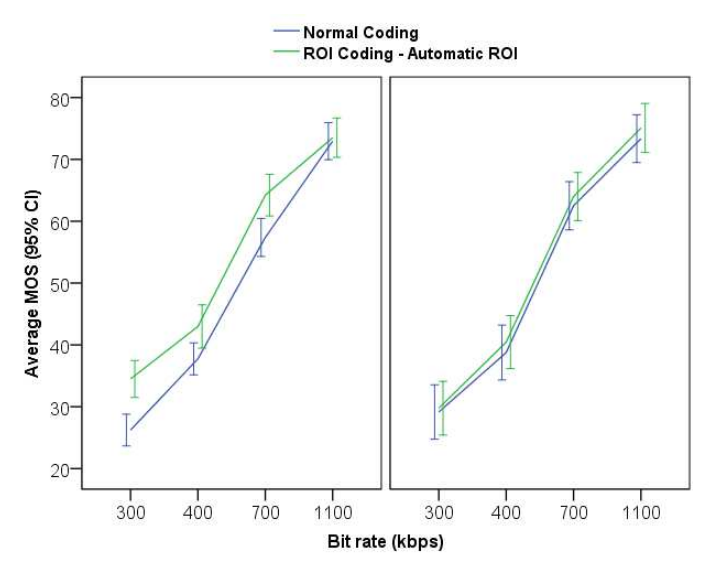

Fig. 10 The mean opinion score for the four video contents watched on the iPhone for different target bit rates in the first session (left figure) and the second session of the study (right figure).

with the same mean. The $t$-value and $p$-value for each bit rate for both the iPhone and iPad are shown in Table 5. At the lower bit rates and up until $700 \mathrm{kbps}$, the perceptual quality of ROI-based videos viewed on the iPhone is significantly higher than the videos with normal coding $(p<.05)$. When these ROI-based videos are viewed on the iPad, the perceptual quality of ROIbased videos viewed is significantly higher than videos with normal coding up to $1100 \mathrm{kbps}(p<.05)$.

\subsubsection{Subjective Evaluation on the iPhone}

Separate analyses were performed for the first session and second session of the user study. Note that the test videos used in the first session of user study consisted of videos $\mathrm{AR}, \mathrm{BW}, \mathrm{CR}$, and $\mathrm{DP}$, and the second session of study used videos AD, BE, DC, and MN. Figure 10 shows that ROI-based video encoding improves the perceptual quality on the iPhone across all the tested bit rates. The improvement of ROI coding over nor-

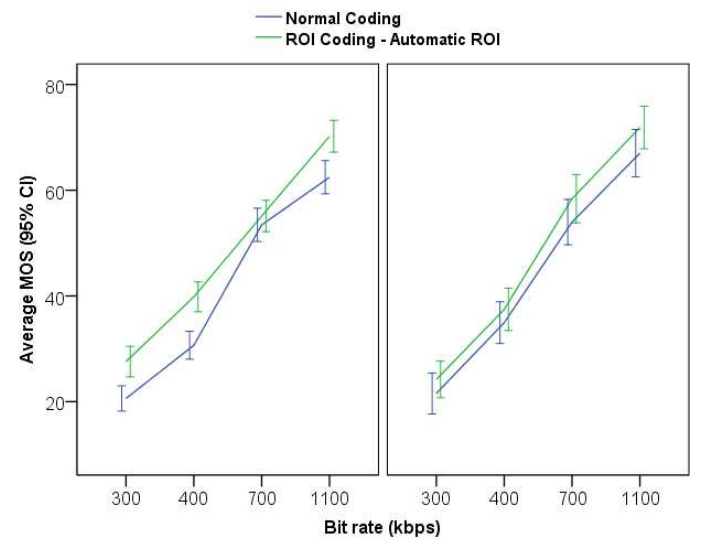

Fig. 11 The mean opinion score for the four video contents watched on the iPad for different target bit rates in the first session (left figure) and the second session of the study (right figure).

mal coding is more apparent in the first round of study compared to what was obtained in the second round of study. This is due to the effect of video contents used in the first and the second round of studies. All videos that were used in second round of studies have the richest textures among the eight videos and two videos (i.e., video $\mathrm{AD}$ and $\mathrm{BE}$ ) have the highest motion among the eight videos as shown in Figure 3. This has an impact on human quality perception (refers to Section 6 for further discussion).

\subsubsection{Subjective Evaluation on the iPad}

There are two key findings from the user study on the iPad. First, on average the quality of ROI-based videos are significantly higher than the normal coding videos when these videos are displayed on the iPad (with a larger resolution and screen size). In other words, when displaying the ROI-based videos at the same video resolution on a large screen, the benefit of ROI coding is still obvious at the higher bit rate (as shown in Figure 
Table 8 Average coding efficiency for ROI coding over normal coding calculated on each video content. Negative (positive) delta rate values indicate a decrease (increase) of bit rate for the same MOS. Negative (positive) delta MOS values indicate a decrease (increase) of MOS for the same bit rate.

\begin{tabular}{|c|c|c|c|c|}
\hline & \multicolumn{2}{|c|}{ iPhone } & \multicolumn{2}{c|}{ iPad } \\
\hline Content & Delta rate $\triangle \mathrm{R}$ & Delta MOS $\triangle \mathrm{D}$ & Delta rate $\triangle \mathrm{R}$ & Delta MOS $\triangle \mathrm{D}$ \\
\hline AR & $-34.5 \%$ & $7.9 \%$ & $-40.6 \%$ & $10.2 \%$ \\
$\mathrm{BW}$ & $-2.8 \%$ & $1.6 \%$ & $-6.3 \%$ & $2.3 \%$ \\
$\mathrm{CR}$ & $-22.8 \%$ & $7.4 \%$ & $-24.2 \%$ & $8.2 \%$ \\
$\mathrm{DP}$ & $-17.3 \%$ & $6.0 \%$ & $-23.5 \%$ & $6.5 \%$ \\
$\mathrm{AD}$ & $-6.4 \%$ & $2.0 \%$ & $-22.5 \%$ & $6.3 \%$ \\
$\mathrm{BE}$ & $-4.6 \%$ & $1.9 \%$ & $-10.9 \%$ & $4.0 \%$ \\
$\mathrm{DC}$ & $0.6 \%$ & $-0.2 \%$ & $-4.8 \%$ & $2.2 \%$ \\
MN & $-6.8 \%$ & $2.5 \%$ & $-10.8 \%$ & $3.5 \%$ \\
\hline Average & $-11.8 \%$ & $3.6 \%$ & $-18.0 \%$ & $5.4 \%$ \\
\hline
\end{tabular}

11 and Table 5). Second, the averaged MOS values are generally lower across bit rates on the iPad compared to the iPhone regardless of coding strategy as shown in Figure 9. This suggests that higher encoding bit rates are needed to provide a better perceived quality for videos viewed on a larger screen.

\subsubsection{Eye Tracking based ROI vs Automatic ROI}

The MOS for ROI-based videos using eye-tracking based ROI is shown in bar plots along with ROI-based videos using automatic ROI and normal coding videos in Figure 9 . It was found that there is no statistically significant perceptual quality difference between using the eye-tracking based ROI and the automatic ROI whenever they are viewed on the iPhone or iPad. A pairedsample t-test in Table 6 shows that there is no statistically significant difference between the two scores $(p>=.05)$, despite the fact that automatic ROI does not completely overlap with the eye-tracking based ROI.

\subsection{Effect of Content Type}

The effect of content type on subject preference is analyzed based on spatial information (SI), colorfulness $(\mathrm{CF})$, and motion vector (MV) metrics. The test videos are classified into three classes (i.e., high, medium, or low) based on the values of those three metrics from Figure 3. Table 7 presents the difference mean opinion score (DMOS) computed as the difference between the scores of ROI-based coding and normal coding averaged across different content and bit rates. The experimental results indicate that videos with content containing low spatial information and medium colorfulness and motion have the highest improvement in perceived quality.

\subsection{Estimation of Bandwidth Saving}

In this study, the average bit rate reduction of ROI coding compared to normal coding for a similar quality is estimated using the Bjøntegaard delta MOS (BDMOS). The BD-MOS uses subjective quality scores instead of PSNR measurements to calculate coding efficiency for similar visual quality $[18,55]$. The SCENIC tool was used for the computation of BD-MOS ${ }^{2}$. Table 8 shows the comparison between ROI coding and normal coding in terms of bit rate reduction for similar MOS (Delta rate $\triangle \mathrm{R}$ ) and subjective quality improvement for similar bit rate (Delta MOS $\triangle \mathrm{D}$ ) for each type of video content. Results based on the subjective scores indicate an average bit rate saving of $11.8 \%$ and $18.0 \%$ for ROI coding relative to normal coding on the iPhone and iPad respectively.

Note that the videos $\mathrm{AR}, \mathrm{CR}$ and DP used in the first round of study provide more bandwidth saving when compared to other videos. In video AR for example, ROI coding achieves $34.5 \%$ and $40.6 \%$ bit rate savings in comparison to normal coding on the iPhone and iPad, respectively. For some content, ROI coding provides smaller bit rate savings. In video BW and DC, ROI coding achieves $6.3 \%$ and $4.8 \%$ bit rate savings on the iPad, respectively.

\section{Discussion}

The subjective evaluation confirms that the ROI-based video encoding improves perceptual quality on the iPhone and iPad for videos encoded in a normal manner with different types of content. The improvement on the iPhone is significantly higher at the lower bit rate range, while at the highest bit rate tested (i.e., $1100 \mathrm{kbps}$ ) no preference could be found for either ROI-based or normal

\footnotetext{
${ }^{2}$ available at https://github.com/phanhart/SCENIC
} 
videos. For an iPad (with a larger resolution and screen size), ROI based video encoding showed superior perceptual quality improvement over normal coding videos encoded up to the tested bit rate of $1100 \mathrm{kbps}$. From the results of this study, it can be seen that neither the use of automatic ROI detection nor the use of eyetracking based ROI that is derived from the human fixation points achieved a statistically significant advantage. This suggests that the use of eye-tracking based ROI for PC monitors is useful for mobile devices.

The average MOS of ROI-based videos for video AR are generally higher compared to other content types across different bit rates. Apart from having the highest automatic ROI detection accuracy (as shown in Table 3), because of lower SI value due to the uniform textured background (i.e., sky), people may tend to focus their attention on salient objects rather than the coding artefacts in the background [33]. For video clips with rich textured backgrounds (higher SI value), the automatic ROI may have difficulty detecting salient objects. Also, from the eye-tracking data, it can be seen in Fig. 5e and $\mathrm{g}$ (video AD and DC, respectively) that fixations are spread over many locations in a frame. For videos of a similar type, the coding artefacts may have more impact on the perceived quality if the automatic ROI does not capture the fixation locations accurately. Note also that, for videos with high motion contents (e.g., video BE), HVS has difficulty perceiving spatial details in fast moving regions and, consequently, viewer may not have sufficient time with which to notice the better quality of ROI. On a positive note, ROI coding is, overall, still perceptually superior over normal coding for these types of content, albeit the improvements are small.

Studies on visual attention modeling $[56,12]$ suggest that different types of distortion affect where people look. In particular, for images distorted by blocking artefacts (compression-type distortions) that are spatially localized, fixations varied depending on the amount and type of distortion. This suggests that the amount of distortion in non-ROI should not exceed a certain level (near-threshold vs suprathreshold) and the quality adjustment may consider the size of ROI/nonROI. This study considers the overall picture quality (both foveal and peripheral vision) of ROI-based videos by means of user study in contrast to experiments in [37], which assumed only the location where people look at as being important in terms of subjective quality.

In order to eliminate the blocking effects, QP values in the transition regions between ROI and non-ROI can be easily adjusted for smoother picture quality. Also, deblocking algorithms can be applied in non-ROI to alleviate coding distortions and improve the overall qual- ity of the video. For example, the non-ROI region can be indicated using the saliency map for deblocking using adaptive bilateral filtering [21].

\section{Conclusion}

This paper investigates the impact of ROI-based video using automatic ROI coding to visually enhance mobile videos. The ROI detection is developed based on a saliency analysis of video frames in combination with face detection, and a concept of emphasizing the center of each frame. The ROI-based video encoding prioritizes the important region to improve the quality of video without increasing the bit rate, at the expense of a balanced approach in reducing the quality of unimportant regions.

Subjective evaluations demonstrate that ROI-based video encoding improves perceived quality of mobile videos across different content types, screen resolutions, and bit rates. The improvement is particularly great in videos encoded at lower bit rates of between 300 to 700 kpbs on the iPhone and up to $1100 \mathrm{kbps}$ on the iPad. The findings from this study are particularly important for video transmission in bandwidth-constrained environments as a means to improve the user experience for mobile users.

Since the allocation of bits for different regions in videos is independent of the algorithms used for encoding the video frames, the proposed ROI prioritization technique may be applied to other existing video codecs (e.g., WebM, HEVC). For future studies, we will study the effect of ROI-based video encoding using the video codec HEVC, newer mobile devices, and in real mobile scenarios.

\section{Acknowledgements}

The authors thank Stefan Winkler for sharing the code to calculate the motion vectors.

\section{References}

1. Akamai: Akamai HD for iPhone encoding best practices. Whitepaper [Online]. Available at http://www.akamai.com/dl/akamai/iphone_wp.pdf (2009)

2. ANSI T1.801.03: Digital transport of one-way video signals - parameters for objective performance assessment. American National Standards Institute, New York (1996)

3. Bian, P., Zhang, L.: Biological plausibility of spectral domain approach for spatiotemporal visual saliency. In: M. Köppen, N. Kasabov, G. Coghill (eds.) Advances in Neuro-Information Processing, Lecture Notes in Computer Science, vol. 5506, pp. 251-258. Springer Berlin / Heidelberg (2009) 
4. Boccignone, G., Marcelli, A., Napoletano, P., Di Fiore, G., Iacovoni, G., Morsa, S.: Bayesian integration of face and low-level cues for foveated video coding. IEEE Transactions on Circuits and Systems for Video Technology 18(12), 1727-1740 (2008)

5. Borji, A., Sihite, D., Itti, L.: Quantitative analysis of human-model agreement in visual saliency modeling: A comparative study. IEEE Transactions on Image Processing 22(1), 55-69 (2013)

6. Cerf, M., Harel, J., Einhäuser, W., Koch, C.: Predicting human gaze using low-level saliency combined with face detection. Advances in neural information processing systems 20, 241-248 (2008)

7. Chan, A.J., Pande, A., Baik, E., Mohapatra, P.: Temporal quality assessment for mobile videos. In: Proceedings of the 18th Annual International Conference on Mobile Computing and Networking, Mobicom 2012 (2012)

8. Chen, M.J., et al.: ROI video coding based on H.263+ with robust skin-color detection technique. IEEE Transactions on Consumer Electronics 49(3), 724-730 (2003)

9. Ciaramello, F.M., Hemami, S.S.: Can you see me now? an objective metric for predicting intelligibility of compressed American sign language video. In: Proceedings of SPIE, Human Vision and Electronic Imaging (2007)

10. De Pessemier, T., De Moor, K., Joseph, W., De Marez, L., Martens, L.: Quantifying subjective quality evaluations for mobile video watching in a semi-living lab context. IEEE Transactions on Broadcasting 58(4), 580-589 (2012)

11. Eichhorn, A., Ni, P.: Pick your layers wisely - a quality assessment of H.264 scalable video coding for mobile devices. In: Proceedings of the IEEE International Conference on Communications, pp. 5446-5451 (2009)

12. Engelke, U., Zepernick, H., Maeder, A.: Visual attention modeling: Region-of-interest versus fixation patterns. In: Picture Coding Symposium, pp. 1-4 (2009)

13. Goldstein, R.B., Woods, R.L., Peli, E.: Where people look when watching movies: Do all viewers look at the same place? Computers in Biology and Medicine 37, 957-964 (2007)

14. Grois, D., Hadar, O.: Recent Advances in Region-ofinterest Video Coding, Recent Advances on Video Coding, chap. 3, pp. 49-76. InTech (2011)

15. Gulliver, S.R., Ghinea, G.: A perceptual comparison of empirical and predictive region-of-interest video. IEEE Transactions on Systems, Man and Cybernetics, Part A: Systems and Humans 39(4), 744-753 (2009). DOI 10. 1109/TSMCA.2009.2019893

16. Guo, C., Zhang, L.: A novel multiresolution spatiotemporal saliency detection model and its applications in image and video compression. IEEE Transactions on Image Processing 19(1), 185-198 (2010)

17. Hadizadeh, H., Bajic, I.: Saliency-aware video compression. IEEE Transactions on Image Processing 23(1), 1933 (2014)

18. Hanhart, P., Ebrahimi, T.: Calculation of average coding efficiency based on subjective quality scores. Journal of Visual Communication and Image Representation 25, $555-564(2014)$

19. Hasler, D., Susstrunk, S.: Measuring colorfulness in natural images. Proceedings of SPIE Human Vision and Electronic Imaging 5007, 87-95 (2003)

20. Himawan, I., Song, W., Tjondronegoro, D.: Automatic region-of-interest detection and prioritisation for visually optimised coding of low bit rate videos. In: Proceedings of IEEE Workshop on Applications of Computer Vision (WACV), pp. 76-82 (2013)
21. Himawan, I., Zele, A.J., Tjondronegoro, D.: Adaptive bilateral filtering using saliency map for deblocking low bit rate videos. In: Proceedings of IEEE International Conference on Multimedia \& Expo, pp. 1-6 (2014)

22. Himawan, I., et al.: Impact of region-of-interest video coding on perceived quality in mobile video. In: Proceedings of IEEE International Conference on Multimedia \& Expo, pp. 79-84 (2012)

23. Itti, L., et al.: A model of saliency-based visual attention for rapid scene analysis. IEEE Transactions on Pattern Analysis and Machine Intelligence 20(11), 12541259 (1998)

24. Jain, E., Sheikh, Y., Shamir, A., Hodgins, J.: Gaze-driven video re-editing. ACM Transactions on Graphics 34 (2015)

25. Jain, E., et al.: Inferring artistic intention in comic art through viewer gaze. In: Proceedings of the ACM Symposium on Applied Perception, pp. 55-62 (2012)

26. Judd, T., Durand, F., Torralba, A.: A benchmark of computational models of saliency to predict human fixations. Tech. rep., Massachusetts Institute of Technology (2012)

27. Jumisko-Pyykkö, S., Hannuksela, M.M.: Does context matter in quality evaluation of mobile television? In: Proceedings of the International Conference on Human Computer Interaction with Mobile Devices and Services, pp. 63-72 (2008)

28. Karlsson, L.S.: Spatio-temporal pre-processing methods for region-of-interest video coding. Ph.D. thesis, Sundsvall, Sweden (2007)

29. Kim, W., et al.: Spatiotemporal saliency detection and its applications in static and dynamic scenes. IEEE Transactions on Circuits and Systems for Video Technology 21(4), $446-456$ (2011)

30. Knoche, H., McCarthy, J.D., Sasse, M.A.: How low can you go? the effect of low resolutions on shot types in mobile TV. Multimedia Tools and Applications 36, 145166 (2008)

31. Koch, C., Ullman, S.: Shifts in selective visual attention: Towards the underlying neural circuity. Human Neurobiology 4, 219-227 (1985)

32. Lai, W., Gu, X.D., Wang, R.H., Ma, W.Y., Zhang, H.J.: A content-based bit allocation model for video streaming. In: IEEE International Conference on Multimedia \& Expo on, vol. 2, pp. 1315-1318 Vol.2 (2004)

33. Larson, E.C., Chandler, D.M.: Most apparent distortion: a dual strategy for full-reference image quality assessment. In: Proceedings of SPIE, Image Quality and System Performance VI. International Society for Optics and Photonics (2009)

34. Le Meur, O., et al.: Do video coding impairments disturb the visual attention deployment? Image Communication 25(8), 597-609 (2010)

35. Lee, J.S., Ebrahimi, T.: Perceptual video compression: A survey. IEEE Journal of Selected Topics in Signal Processing 6(6), 684-697 (2012)

36. Lee, J.S., et al.: Subjective quality evaluation of foveated video coding using audio-visual focus of attention. IEEE Journal of Selected Topics in Signal Processing 5(7), 1322-1331 (2011)

37. Li, Z., Qin, S., Itti, L.: Visual attention guided bit allocation in video compression. Image and Vision Computing 29(1), 1 - 14 (2011)

38. Liu, F., Gleicher, M.: Video retargeting: automating pan and scan. In: Proc. ACM Multimedia, pp. 241-250. Santa Barbara, USA (2006)

39. Liu, Y., Li, Z.G., Soh, Y.C.: Region-of-interest based resource allocation for conversational video communication 
of H.264/AVC. IEEE Transactions on Circuits and Systems for Video Technology 18(1), 134-139 (2008)

40. Lu, T., Yuan, Z., Huang, Y., Wu, D., Yu, H.: Video retargeting with nonlinear spatial-temporal saliency fusion. In: Proc. IEEE International Conference on Image Processing, pp. $1801-1804$ (2010)

41. Marius't Hart, B., et al.: Gaze allocation in natural stimuli: Comparing free exploration to head-fixed viewing conditions. Visual Cognition 17, 1132-1158 (2009)

42. McCarthy, J.D., Sasse, M.A., Miras, D.: Sharp or smooth?: Comparing the effects of quantization vs. frame rate for streamed video. In: Proc. SIGCHI Conference on Human Factors in Computing Systems, pp. 535-542 (2004)

43. Mital, P.K., Smith, T.J., Hill, R., Henderson, J.M.: Clustering of gaze during dynamic scene viewing is predicted by motion. Cognitive Computation 3, 5-24 (2011)

44. Moorthy, A., Choi, L.K., Bovik, A., de Veciana, G.: Video quality assessment on mobile devices: Subjective, behavioral and objective studies. IEEE Journal of Selected Topics in Signal Processing 6(6), 652-671 (2012)

45. Nyström, M., Holmqvist, K.: Effect of compressed offline foveated video on viewing behavior and subjective quality. ACM Trans. Multimedia Computing, Communications, Applications 6(1) (2010)

46. Oeldorf-Hirsch, A., Donner, J., Cutrell, E.: How bad is good enough?: Exploring mobile video quality trade-offs for bandwidth-constrained consumers. In: Proceedings of the 7th Nordic Conference on Human-Computer Interaction: Making Sense Through Design, pp. 49-58 (2012)

47. O'Shea, R.P.: Thumb's rule tested: visual angle of thumb's width is about 2 deg. Perception 20(3), 415418 (1991)

48. Ries, M.: Video quality estimation for mobile video streaming. Ph.D. thesis, Vienna University of Technology (2008)

49. Ries, M., Nemethova, O., Rupp, M.: Performance evaluation of mobile video quality estimators. In: Proceedings of the European Signal Processing Conference (2007)

50. Sangwine, S.: The discrete quaternion Fourier transform. In: Proceedings of International Conference on Image Processing and Its Applications, vol. 2, pp. $790-793$ vol.2 (1997)

51. Sheikh, H.R., Evans, B.L., Bovik, A.C.: Real-time foveation techniques for low bit rate video coding. RealTime Imaging 9(1), 27-40 (2003)

52. Song, W., Tjondronegoro, D., Docherty, M.: Saving bitrate vs. pleasing users: Where is the break-even point in mobile video quality? In: Proceedings of the 19th ACM International Conference on Multimedia, pp. 403$412(2011)$

53. Song, W., et al.: Impact of zooming and enhancing region of interest for optimizing user experience on mobile sports video. In: Proceedings of ACM International Conference on Multimedia (2010)

54. Tsapatsoulis, N., Rapantzikos, K., Pattichis, P.C.: An embedded saliency map estimator scheme: Application to video encoding. International Journal of Neural Systems 17, 289-304 (2007)

55. Řréábek, M., Ebrahimi, T.: Comparison of compression efficiency between HEVC/H. 265 and VP9 based on subjective assessments. In: Proceedings of SPIE Optical Engineering + Applications (2014)

56. Vu, C.T., Larson, E., Chandler, D.: Visual fixation patterns when judging image quality: Effects of distortion type, amount, and subject experience. In: IEEE Southwest Symposium on Image Analysis and Interpretation, pp. $73-76(2008)$
57. Wang, Z., Bovik, A., Sheikh, H., Simoncelli, E.: Image quality assessment: from error visibility to structural similarity. IEEE Transactions on Image Processing 13(4), 600-612 (2004)

58. Wang, Z., Lu, L., Bovik, A.: Foveation scalable video coding with automatic fixation selection. IEEE Transactions on Image Processing 12(2), 243-254 (2003)

59. Wiegand, T., Sullivan, G., Luthra, A.: Draft ITU-T recommendation and final draft international standard of joint video specification (ITU-T Rec. H.264 - ISO/IEC 14496-10 AVC). Joint Video Team (JVT) of ISO/IEC MPEG and ITU-T VCEG, JVT - G050r1 (2003)

60. Winkler, S.: Analysis of public image and video databases for quality assessment. IEEE Journal of Selected Topics in Signal Processing 6(6), 616-625 (2012)

61. Wu, H.R., Reibman, A., Lin, W., Pereira, F., Hemami, S.: Perceptual visual signal compression and transmission. Proceedings of the IEEE 101(9), 2025-2043 (2013)

62. Yang, X., et al.: Rate control for videophone using local perceptual cues. IEEE Transactions on Circuits and Systems for Video Technology 15(4), 496-507 (2005)

63. You, W., Sabirin, M.S.H., Kim, M.: Real-time detection and tracking of multiple objects with partial decoding in H.264/AVC bitstream domain. In: Proceedings of SPIE Real-Time Image and Video Processing (2009) 